Onkologe 2021 $27: 691-696$

https://doi.org/10.1007/s00761-021-00972-1

Angenommen: 6. Mai 2021

Online publiziert: 17. Mai 2021

(c) Der/die Autor(en) 2021

Marie von Lilienfeld-Toal' $\cdot$ Christina Rieger $^{2} \cdot$ Nicola Giesen $^{3}$ - Bernhard Wörmann ${ }^{4}$

'Schwerpunkt Infektionen in der Hämatologie und Onkologie, Klinik für Innere Medizin II, Abteilung für Hämatologie und Internistische Onkologie, Universitätsklinikum Jena, Jena, Deutschland

${ }^{2}$ Hämato-Onkologie Germering, Schwerpunktpraxis des Tumorzentrums München, Germering, Deutschland

${ }^{3}$ Abteilung für Hämatologie und Onkologie, Innere Medizin V, Universitätsklinikum Heidelberg, Heidelberg, Deutschland

${ }^{4}$ Medizinische Klinik mit Schwerpunkt Hämatologie, Onkologie und Tumorimmunologie, Charité Universitätsmedizin Berlin, Berlin, Deutschland

\title{
Impfung gegen SARS-CoV-2 bei Krebspatienten
}

den durch antigenpräsentierende Zellen aufgenommen und führen in Lymphknoten $\mathrm{zu}$ einer adaptiven Immunantwort mit der Bildung neutralisierender Antikörper sowie spezifischer T-Zellen. Es gibt zwei verschiedene Wege, die VirusmRNA in die menschlichen Zellen zu bringen: die so genannten mRNA-Impfstoffe enthalten mit Trägermolekülen umhüllte mRNA. Diese Trägermoleküle (häufig Fettmoleküle beziehungsweise Nanopartikel) stabilisieren die sonst sehr leicht zerfallende RNA, sodass sie von den menschlichen Zellen aufgenommen und umgeschrieben werden kann. Im Gegensatz dazu benutzen die Vektorimpfstoffe einen viralen Vektor, in der Regel rekombinationsdefiziente Adenoviren, der DNA enthält. Vektor und Inhalt werden in die menschliche Zelle aufgenommen, die DNA wird im Zellkern zu RNA umgeschrieben, und der weitere Werdegang ist im Wesentlichen wie bei den mRNA-Impfstoffen.

Da die viralen Vektoren aus rekombinationsdefizienten Viren hergestellt werden, die einen Enzymdefekt haben, der ihnen ein Wachstum nur in entsprechend substituiertem Medium erlaubt, gelten diese Impfstoffe als Totimpfstoffe. Auch die mRNA-Impfstoffe sind Totimpfstoffe, da sie keinerlei lebendes Material enthalten. Beide Impfstofftypen sind nicht als Gentherapie anzusehen, da die mRNA-Impfstoffe vollkommen im Zytoplasma verbleiben und nicht mit dem menschlichen Genom in Berührung kommen. Die DNA der Vektorimpfstoffe wird zwar im Zellkern umgeschrieben, aber nicht in das menschliche Genom integriert und kann auch nicht weitergegeben werden. Somit erfolgt auch hier keine Gentherapie (• Abb. 1).

\section{Wirksamkeit der zugelassenen COVID-19-Impfstoffe}

Bisher sind 4 Impfstoffe in der EU zugelassen. Die Daten dieser Zulassungsstudien sind publiziert und/oder in den Zulassungsunterlagen hinterlegt [8]. Charakteristika der Impfstoffe und Ergebnisse zur Wirksamkeit sind in $\bullet$ Abb. 2 zusammengefasst.

Die häufigsten Nebenwirkungen sind eine Lokalreaktion an der Injektionsstelle, die vor allem mit Schmerzen einhergeht. Systemische Nebenwirkungen wie Fatigue, Kopfschmerzen oder Fieber treten ebenfalls bei etwa der Hälfte der Geimpften auf. Interessanterweise erleiden vor allem jüngere und weibliche Menschen Nebenwirkungen. Nach Verabreichung der mRNA-Impfstoffe sind die Nebenwirkungen nach der zweiten Injektion ausgeprägter als nach der ersten, während dies nach Verabreichung der AstraZeneca-Vektorvakzine umgekehrt der Fall ist. Sehr selten treten nach den mRNA-Impfstoffen anaphylaktische Reaktionen auf. Diese traten bei der Mehrheit der Betroffenen innerhalb von $15 \mathrm{~min}$ nach der Injektion auf. Deshalb sollte nach der Verabreichung des Impfstoffs immer eine angemessene medizinische Versorgung und Überwacoronavirus-infektion-covid-19-bei-patientinnen-mit-blut-und-krebserkrankungen. 


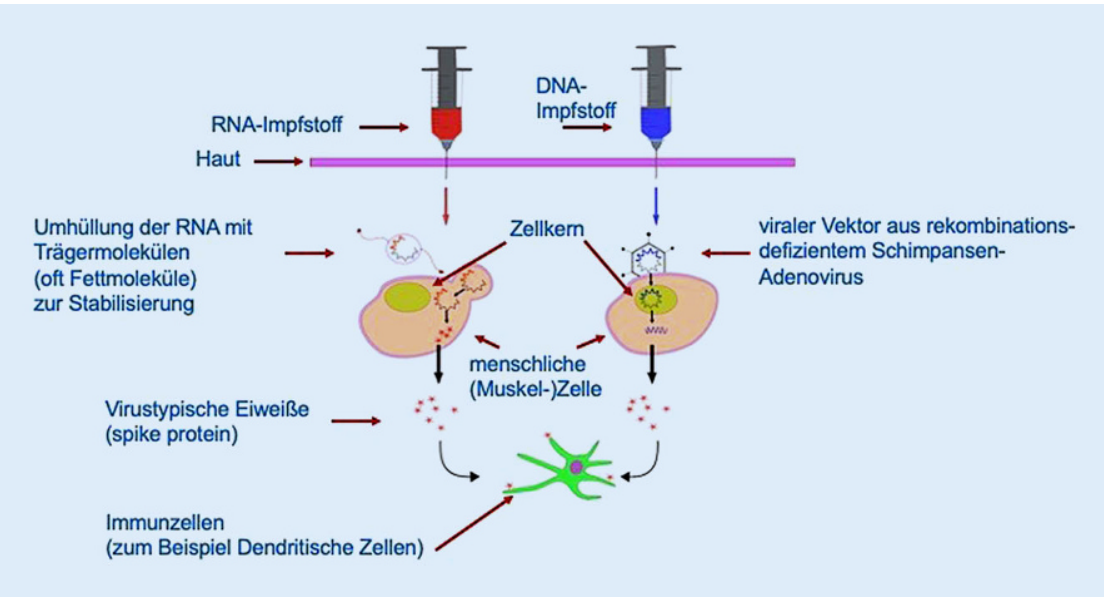

Abb. 1 ॥ Funktionsweise der Impfstoffe (c/o Mai Hoang)

chung bereitstehen. Nach der Impfung wird eine engmaschige Beobachtung von mindestens $15 \mathrm{~min}$ empfohlen. Eine zweite Dosis des Impfstoffs sollte nicht an Personen verabreicht werden, bei denen eine Anaphylaxie nach der ersten Dosis aufgetreten ist.

Im zeitlichen Zusammenhang mit der Applikation der Vektorvakzinen zur COVID-19-Schutzimpfung sind Sinusund Hirnvenenthrombosen (CVST) mit systemischen Gerinnungsstörungen und z.T. schweren klinischen, auch tödlichen Verläufen beobachtet worden [3, $5,14,16]$. Beispielsweise wurde sehr selten nach einer Impfung mit Vaxzevria von AstraZeneca eine Kombination von Thrombose und Thrombozytopenie, in einigen Fällen einhergehend mit Blutungen, beobachtet. Dies schließt schwere Fälle ein, die sich als venöse Thrombose präsentierten, einschließlich des Auftretens in ungewöhnlichen Bereichen, wie zum Beispiel zerebrale Sinusvenenthrombose, Mesenterialvenenthrombose sowie arterielle Thrombose, bei gleichzeitiger Thrombozytopenie. Die meisten dieser Fälle traten innerhalb der ersten sieben bis vierzehn Tage nach der Impfung und bei Frauen unter 55 Jahren auf, was möglicherweise auf die verstärkte Anwendung des Impfstoffs in dieser Bevölkerungsgruppe zurückzuführen ist. Einige Fälle hatten einen tödlichen Ausgang [5]. Weltweit wurde unterschiedlich reagiert. Diese schweren Gerinnungsstörungen sind sehr selten, die genaue Häufigkeit ist unklar. Frauen $<55$ Jahren sind häufiger betroffen. Es gibt bis- her keine Hinweise, dass Patient ${ }^{\star}$ innen mit vorbestehenden, hereditären oder erworbenen Gerinnungsstörungen ein erhöhtes Risiko für diese Komplikationen haben. Derzeit werden für diese Komplikation die Begriffe „vaccine-induced immune thrombotic thrombocytopenia“ (VITT) oder vakzineinduzierte prothrombotische Immunthrombozytopenie (VIPIT) gebraucht. Ein möglicher Pathomechanismus ist die Induktion einer Antikörperbildung gegen Thrombozytenantigene im Rahmen der vakzineinduzierten Immunstimulation. Diese Antikörper induzieren eine Thrombozytenaktivierung über den Fc-Rezeptor in Analogie zur heparininduzierten Thrombozytopenie (HIT). Die Gesellschaft für Thrombose- und Hämostaseforschung (GTH) und andere Fachgesellschaften haben Empfehlungen für die Diagnostik bei vorliegendem Verdacht und für den Umgang mit diesen Gerinnungsstörungen publiziert [9]. Gerinnungsstörungen in der Vorgeschichte einschl. Thrombosen sind keine Kontraindikation gegen eine Impfung mit der AstraZenecaVakzine. Während seitens der EMA und der WHO aktuell keine Einschränkungen des Einsatzes der AstraZenecaVakzine formuliert wurden, empfiehlt in Deutschland die STIKO den Einsatz der AstraZeneca-Vakzine nun primär für Personen $\geq 60$ Jahren, wobei nach entsprechender Aufklärung und individueller Risiko-Nutzen-Abwägung weiterhin auch der Einsatz bei jüngeren Patienten möglich bleibt.
Neben den in - Abb. 2 zusammengefassten Daten der Zulassungsstudien sind auch Erfahrungen aus der Versorgung (Real World Data) relevant. Dabei zeigte sich im schottischen Register mit 5,4 Mio. Personen bereits nach der ersten Schutzimpfung mit der AstraZenecaVakzine ein Rückgang in der COVID-19bezogenen Hospitalisationsrate um $95 \%$ [17].

Inzwischen wurden in den Medien auch Ergebnisse einer Interimsanalyse der erweiterten Zulassungsstudie bei Personen in den USA, Chile und Peru berichtet. Sie bestätigen die Daten der $\mathrm{Zu}$ lassung, sind aber bisher nicht voll in einem Peer-Review-Journal publiziert.

Weltweit gibt es eine Reihe weiterer Impfstoffe. Dazu gehören u. a. der Impfstoff der Fa. Sinovac Biotech, Sputnik V und der Impfstoff der Fa. CureVac [7]. Sie sind bisher nicht für die EU zugelassen. Bisher ist nicht vollständig geklärt, inwieweit die zugelassenen Schutzimpfungen auch gegen mutierte Viren (,variants of concern") wirksam sind. Die bisher vorliegenden Daten zeigen, dass die für die EU zugelassenen Impfstoffe auch gegenüber der aktuell (Stand 04/2021) in Deutschland dominanten Variante B.1.1.7. einen wirksamen Schutz bieten, was aber möglicherweise nicht auf alle existierenden Varianten zutrifft $[4,20]$.

\section{Empfehlungen zur Schutz- impfung gegen COVID-19 bei Krebspatient*innen}

Krebspatient*innen und Menschen mit Immunsuppression aufgrund hämatologischer Erkrankungen sowie das betreuende medizinische Personal werden in Deutschland wegen des erhöhten Risikos für einen schweren Verlauf in eine hohe Prioritätsstufe für die Impfung eingeordnet. Auch bis zu 2 enge Kontaktpersonen aus dem Angehörigenkreis pflegebedürftiger Krebspatient ${ }^{\star}$ innen haben eine erhöhte Priorität für die Impfung. Es liegen trotz der weltweit millionenfachen Impfungen gegen COVID-19 bisher für die hier betrachtete Gruppe der Patient ${ }^{*}$ innen mit Krebserkrankungen nur zahlenmäßig begrenzte und vorläufige Daten vor, sodass Entscheidungen und Empfehlungen teilweise auf Analogien 
zu Impfungen gegen Influenza bei dieser Patientengruppe zurückgreifen.

\section{Zeitpunkt der Impfung}

Der Schutz einer COVID-19-Impfung kann bei immunsupprimierten $\mathrm{Pa}$ tient*innen geringer sein, erste Daten deuten auf eine erniedrigte Antikörperbildung nach einer einmaligen Impfung hin [8]. Umfassende Daten liegen dazu nicht vor, auch nicht zu einer schädlichen Wirkung der Impfstoffe. In der praktischen Versorgung empfehlen wir, folgende Aspekte zu berücksichtigen:

\section{Geplante Operation oder Bestrahlung}

- Wenn immer möglich, sollte die Schutzimpfung vor Beginn einer onkologischen Therapie erfolgen. Das betrifft auch chirurgische Eingriffe oder eine Bestrahlung.

\section{Chemotherapie}

- Wenn immer möglich, sollte die Schutzimpfung vor Beginn einer systemischen Therapie erfolgen.

- Während einer laufenden zyklischen Chemotherapie sollte versucht werden, einen zeitlichen Abstand zur systemischen Therapie zu erreichen, um das Risiko überlappender Nebenwirkungen zu vermindern. Daten prospektiver Studien zum optimalen Zeitpunkt der COVID19-Schutzimpfung während einer systemischen Therapie liegen nicht vor. Ein Aussetzen oder Verschieben der Chemotherapie wird nicht routinemäßig empfohlen. Daten zur Influenza-Schutzimpfung mit Booster-Impfungen zeigen, dass der Abstand zur Chemotherapie hinsichtlich der Impfwirkung keine wesentliche Rolle spielt [11].

\section{Immunsuppressive Therapie}

- Auch unter einer fortlaufenden immunsuppressiven Therapie gibt es keinen optimalen Zeitpunkt für die Schutzimpfung. Ein Aussetzen der Therapie wird nicht empfohlen. Analog zur Chemotherapie ist in der Regel bei einer Impfung mit

Onkologe 2021 · 27:691-696 https://doi.org/10.1007/s00761-021-00972-1

(c) Der/die Autor(en) 2021

\section{M. von Lilienfeld-Toal · C. Rieger · N. Giesen · B. Wörmann}

\section{Impfung gegen SARS-CoV-2 bei Krebspatienten}

\section{Zusammenfassung}

Patient*innen mit Krebserkrankungen haben ein erhöhtes Risiko, schwere Verläufe einer COVID-19-Erkrankung zu erleiden. Spezifische Schutzmaßnahmen inkl. der COVID-19Impfungen sind daher hier von besonderer Bedeutung. Eine durch Therapie und Grunderkrankung oftmals bedingte Abwehrschwäche kann jedoch eine Herausforderung für Impfstrategien bei diesen Patient ${ }^{*}$ innen darstellen. Aktuell sind in Deutschland vier COVID-19-Impfstoffe zugelassen, zwei mRNA- und zwei vektorbasierte Impfstoffe, die alle eine sehr gute Wirksamkeit gegen schwere Krankheitsverläufe zeigen. Hinsichtlich des Wirkmechanismus ist allen vier Impfstoffen die Induktion einer Produktion virusspezifischer Proteine in menschlichen Zellen gemeinsam mit nachfolgender Aktivierung einer adaptiven Immunantwort. Für Krebspatient*innen und medizinisches Personal wird die Impfung priorisiert empfohlen. Ein optimaler Zeitpunkt für die Impfung bei Neuerkrankten scheint vor
Beginn einer Tumortherapie zu sein. Aufgrund des erhöhten Risikos von Krebspatient*innen für schwere Verläufe von COVID-19 wird die Impfung jedoch auch unter laufender Tumortherapie empfohlen. Die Impfantwort ist hier allerdings möglicherweise reduziert. In den besonderen Konstellationen einer vorherigen Stammzelltransplantation oder einer B-Zell-depletierenden Therapie wird nach Möglichkeit ein mehrmonatiger Abstand zwischen Therapie und Impfung empfohlen, da hier ansonsten mit einer deutlich reduzierten Impfantwort gerechnet werden muss. Wenn sich erste Hinweise auf nur eine geringe Serokonversion bei Krebspatient*innen nach einmaliger Impfung bestätigen, können zukünftige Empfehlungen in Richtung mehrfacher Impfungen bei diesen Patient*innen gehen.

\section{Schlüsselwörter}

Immunsuppression · COVID-19 - mRNA Impfstoff · Vektor-Impfstoff · Prävention

\section{Vaccination against SARS-CoV-2 in cancer patients}

\section{Abstract}

Patients with cancer are at an increased risk to suffer severe coronavirus disease 2019 (COVID-19). Therefore, specific preventative measures including COVID19 vaccines are especially important. Both anticancer therapies and the underlying malignancy itself can lead to significant immunosuppression posing a particular challenge for vaccination strategies in these patients. At the moment, four COVID-19 vaccines are European Medicines Agency (EMA) approved in Germany: two mRNA and two viral vector-based vaccines. All four vaccines show excellent protection against severe COVID-19. Their mechanism of action relies on the induction of the production of virus-specific proteins by human cells and the following activation of a specific adaptive immune response. Vaccination against COVID-19 has been prioritized for cancer patients and medical personnel in Germany. Regarding timing of vaccination, vaccination prior to initiation of anticancer therapy seems ideal in newly diagnosed disease. However, due to the significant risk of severe COVID19 in cancer patients, vaccination is also strongly recommended for patients already undergoing anticancer therapy. In these patients, immune response might be reduced. In two particular patient cohorts, namely stem cell transplant recipients and patients treated with B-cell depleting agents, an interval of several months following therapy is recommended because otherwise the response to vaccination will most likely be severely reduced. Preliminary data suggest only low rates of seroconversion following a single shot of vaccine in cancer patients. Therefore, on the long run, repeat vaccination regimens might be preferable in cancer patients.

\section{Keywords} Immunosuppression · COVID-19 · mRNA vaccine $\cdot$ vector-vaccine p prevention 


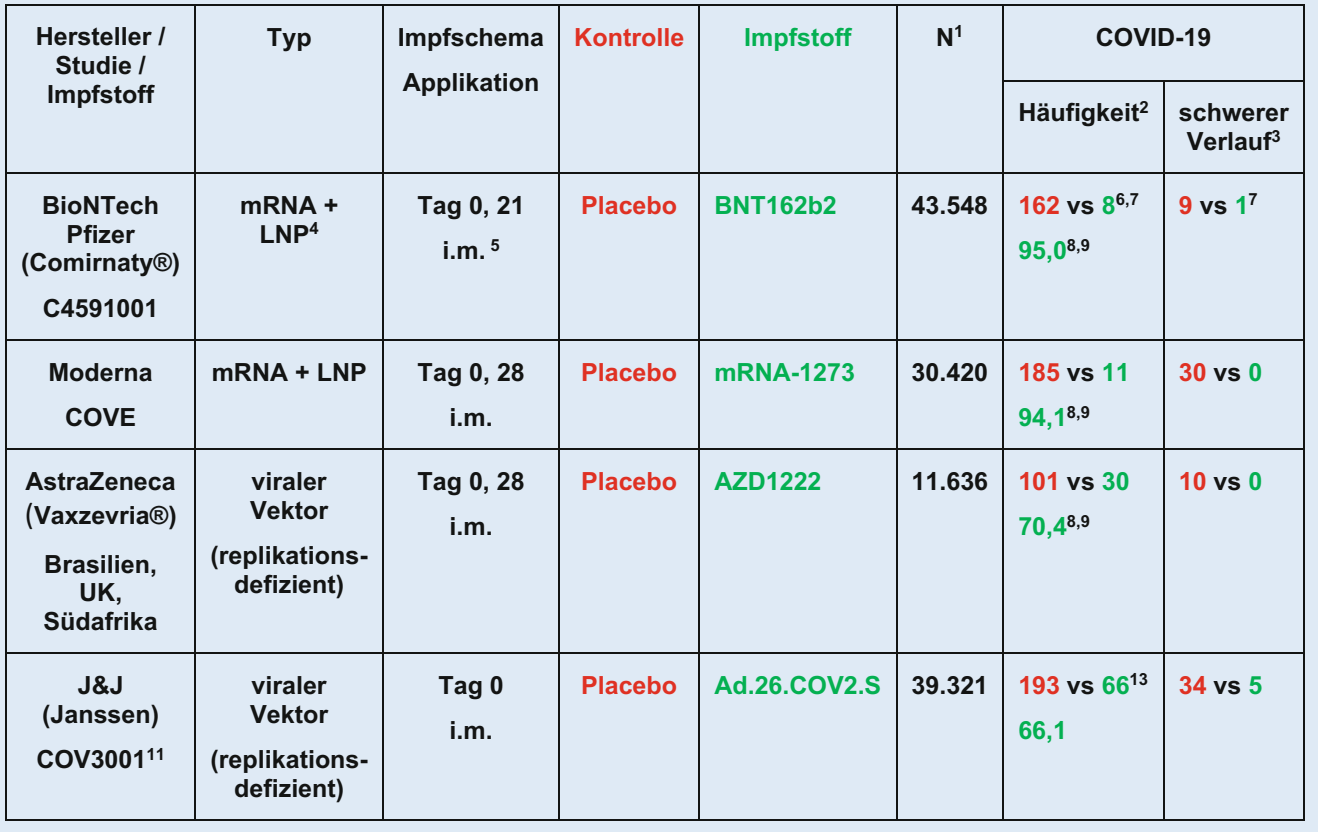

N - Anzahl Probanden; ${ }^{2}$ Häufigkeit - Anzahl infizierter Personen mit Symptomen; ${ }^{3}$ schwerer Verlauf - Anzahl Patient*innen mit schwerer Verlaufsform von COVID-19, Anzahl hospitalisierter Patient*innen ; ${ }^{4}$ Typ: mRNA - Messenger RNA; LNP - Lipid Nanopartikel; ${ }^{3}$ i.m. - intramuskulär; ${ }^{6}$ Anzahl infizierter Personen; ${ }^{7}$ Ergebnis für Kontrolle, Ergebnis für Neue Therapie; 8 Wirksamkeit für Neue Therapie; ${ }^{9}$ Wirksamkeit in \%; ${ }^{10}$ Randomisierung Verum vs Placebo 3:1, die anderen Zulassungsstudien hatten eine 1:1 Randomisierung; ${ }^{11}$ Daten nicht in Peer-Review-Journal publiziert;
Abb. $2<$ In der EU zugelassene COVID-19-Vakzine - publizierte Daten zur Wirksamkeit
Booster von einer guten Impfantwort auszugehen.

- Eine Ausnahme bildet die B-Zelldepletierende Therapie mit AntiCD-20-Antikörpern, bispezifischen Antikörpern, Antikörperkonjugaten, Anti-CD19-CAR-T-Zellen oder BTKi (Bruton-Tyrosinkinase-Inhibitoren)/bcl2-Inhibitoren („B-cell lymphoma 2 “). Hier ist zu erwarten, dass - in Analogie zur InfluenzaSchutzimpfung - die Impfantwort trotz Booster wahrscheinlich deutlich reduziert ist. Hierfür sprechen Daten einer deutlich erniedrigten Serokonversion bei diesen Patienten [6]. Daher kann ein zeitlicher Abstand von mindestens 3 Monaten oder sogar länger zur letzten Therapie erwogen werden. In anderen Empfehlungen wird sogar ein Abstand von bis zu 6 Monaten empfohlen. Allerdings beruhen diese Empfehlungen auf der Annahme, dass möglicherweise kein wirksamer Schutz aufgebaut wird und nicht auf einer schädlichen Wirkung. Da der Schutz durch die COVID-19-Vakzine jedoch auch T-Zell-vermittelt ist
[13], stellt die B-Zell-Depletion an sich keine absolute Kontraindikation für die COVID-19-Schutzimpfung dar, und es kann im Einzelfall sinnvoll sein, trotz fehlender B-Zellen in einer Hochrisikokonstellation zu impfen. Aufgrund des verminderten Ansprechens sollten diese Patient*innen ausdrücklich auf das weitere Einhalten der Hygieneregeln hingewiesen werden.

Auch Patient*innen vor oder nach einer allogenen Stammzelltransplantation bilden eine eigene Gruppe. Die COVID-19-assoziierte Mortalität ist nach einer allogenen Stammzelltransplantation möglicherweise erhöht [15]. Deshalb sollte, wenn immer möglich, die Schutzimpfung vor Beginn einer systemischen Therapie erfolgen. Zur Schutzimpfung nach einer allogenen Stammzelltransplantation liegen Empfehlungen der European Society for Blood and Marrow Transplantation vor (https://www.ebmt.org/covid-19and-bmt). Diese beinhalten einen Abstand von mindestens 3 Monaten nach der Transplantation sowie eine
Verzögerung der Schutzimpfung bei unkontrollierter Graft-versus-HostDisease, nach B-Zell-depletierender oder nach CAR-T-Zell-Therapie sowie nach Therapie mit ATG oder Alemtuzumab.

Bei der patientenindividuellen Entscheidungsfindung über die Durchführung einer COVID-19-Impfung gelten die Grundsätze des Shared Decision Making zwischen Arzt und Patient*in unter besonderer Berücksichtigung der individuellen Risikosituation.

Die bisher zugelassenen Impfstoffe werden intramuskulär appliziert. Eine subkutane Applikation kann die Wirksamkeit beeinträchtigen und wird daher nicht empfohlen. Bei Patient ${ }^{*}$ innen mit klinischer manifester Blutungsneigung (Thrombozytopenie, Antikoagulation o.a.) wird eine ausreichend lange Kompression an der Injektionsstelle und gegebenenfalls das Anliegen eines Stauschlauchs für 2-3 min nach der Injektion empfohlen. Ein Aussetzen der Antikoagulation wird nicht empfohlen, bei ausgeprägter Thrombozytopenie 
kann die Gabe eines Thrombozytenkonzentrats erwogen werden.

Bei Patient*innen mit der Vorgeschichte einer anaphylaktischen Reaktion soll das Risiko einer schweren Nebenwirkung besonders sorgfältig gegenüber dem erwarteten Nutzen abgewogen werden. Grundsätzlich wird für diese Patienten ein längeres Nachbeobachtungsintervall nach der Impfung in der Impfpraxis/dem Impfzentrum empfohlen.

Die zuletzt beschriebenen thromboembolischen Ereignisse in Zusammenhang mit vektorbasierten Impfstoffen (VITT, VIPIT, s. oben) sind unabhängig von Tumorerkrankungen aufgetreten. Bisher liegen keine Hinweise vor, dass diese Komplikationen bei Patient ${ }^{*}$ innen mit Krebserkrankungen häufiger vorkommen.

Mit Ausnahme der Vakzine von Johnson \& Johnson ist für alle bisher in der EU zugelassenen Impfstoffe eine zweite Impfung vorgesehen. In den Zulassungsstudien erfolgte diese nach 21 bzw. 28 Tagen (• Abb. 2). Aufgrund der aktuellen Engpasssituation in der Impfstoffverfügbarkeit wird das Intervall bis zur zweiten Impfung häufig verlängert, auf bis zu 12 Wochen.

Daten einer aktuellen vergleichenden Studie zu BNT162b2 (BioNTech/Pfizer) deuten darauf hin, dass die Serokonversion bei Krebspatient*innen nach der ersten Schutzimpfung deutlich geringer als bei Gesunden ausfällt mit diesen Raten: $40 \%$ bei Patient*innen mit soliden Tumoren, $15 \%$ bei hämatologischen Neoplasien, $>90 \%$ bei Gesunden. Nach der zweiten Impfung wurde bei $\mathrm{Pa}$ tient ${ }^{*}$ innen mit soliden Tumoren eine Serokonversion von $95 \%$ erreicht [8]. Niedrigere Serokonversionsraten nach einmaliger Impfung wurden auch bei Z.n. Organtransplantation beobachtet [2]. Diese Daten deuten darauf hin, dass bei Verwendung eines mRNA-Impfstoffs die zweite Schutzimpfung bei Krebspatient*innen zeitnah, d.h. nach 3 bzw. 4 Wochen, stattfinden sollte. Bei vektorbasierten Impfstoffen kann ein längeres Intervall sinnvoll sein.

Möglicherweise ist nach stattgehabter Impfung von ausgeprägt immunsupprimierten Patient ${ }^{*}$ innen nach Regenerati- on eine spätere, erneute Schutzimpfung sinnvoll. Eine Empfehlung zu dieser Indikation wird erst nach Vorliegen weiterer Daten möglich sein. Offen ist auch, ob bei Patient ${ }^{*}$ innen nach durchgemachter COVID-19-Erkrankung eine einmalige Impfung zur Boosterung entsprechend der aktuellen STIKO-Empfehlung ausreicht oder ob eher eine vollständige Schutzimpfung erforderlich ist.

\section{Wahl des Impfstoffs bei Krebspatient*innen}

Die Wirksamkeit der beiden mRNA-basierten Impfstoffe ist etwa gleich hoch. Unterschiede finden sich vor allem in der Lagerung, der Applikation und im Nebenwirkungsspektrum. Die vektorbasierten Impfstoffe zeigen eine etwas geringere Wirksamkeit im Schutz vor Infektionen, siehe - Abb. 2. Allerdings ist der Schutz vor schweren Verläufen von COVID-19 bei den zugelassenen Impfstoffen gleich hoch. Es gibt bisher auch keine Hinweise auf eine höhere Rate von Nebenwirkungen bei Krebspatient*innen nach der Schutzimpfung.

Entsprechend gibt es bisher keine Evidenz für Empfehlungen für oder gegen einen der zugelassenen Impfstoffe. Derzeit ist jeder Impfstoff besser als kein Impfstoff. Allerdings suggerieren die o. a. Daten, dass eine Einmal-Schutzimpfung bei Krebspatient*innen nicht ausreichend ist.

Langzeitergebnisse zur Dauer der Wirksamkeit der Impfung und zur Sicherheit liegen für keinen der Impfstoffe vor.

\section{Fazit für die Praxis}

- Eine COVID-19-Schutzimpfung sollte routinemäßig allen Krebspatient*innen angeboten werden.

- Neben einer bekannten Anaphylaxie gibt es praktisch keine Kontraindikationen gegen die Impfung, wenn auch teilweise von einer reduzierten Immunantwort ausgegangen werden muss.

- Ungefährliche Nebenwirkungen wie lokale Reaktionen und Fatigue sind häufig und vor allem bei jüngeren Frauen zu erwarten, wobei die mRNA-
Impfstoffe vor allem nach der 2. und der Vektorimpfstoff vor allem nach der 1. Injektion Nebenwirkungen hervorruft.

- Bei Krebspatient*innen sind Impfregime mit Doppelimpfung aufgrund des erwarteten besseren Ansprechens zu bevorzugen.

\section{Korrespondenzadresse}

Prof. Dr. Marie von Lilienfeld-Toal

Schwerpunkt Infektionen in der Hämatologie und Onkologie, Klinik für Innere Medizin II, Abteilung für Hämatologie und Internistische Onkologie, Universitätsklinikum Jena Am Klinikum 1, 07747 Jena, Deutschland Marie.von_Lilienfeld-Toal@med.uni-jena.de

Funding. Open Access funding enabled and organized by Projekt DEAL.

\section{Einhaltung ethischer Richtlinien}

Interessenkonflikt. M. von Lilienfeld-Toal erhielt Honorare bzw. Forschungsförderung von Janssen Cilag, Celgene, BMS, medac, Gilead, Pfizer, Takeda, abbvie, AstraZeneca, BioNTech, Oncopeptides, Novartis, gsk, Shionogi, Amgen, BMBF und Deutsche Krebshilfe. C. Rieger erhielt Honorare von AstraZeneca und Pfizer N. Giesen erhielt Honorare von MSD und Pfizer. B. Wörmann gibt an, dass kein Interessenkonflikt besteht.

Für diesen Beitrag wurden von den Autoren keine Studien an Menschen oder Tieren durchgeführt. Für die aufgeführten Studien gelten die jeweils dort angegebenen ethischen Richtlinien.

Open Access. Dieser Artikel wird unter der Creative Commons Namensnennung 4.0 International Lizenz veröffentlicht, welche die Nutzung, Vervielfältigung, Bearbeitung, Verbreitung und Wiedergabe in jeglichem Medium und Format erlaubt, sofern Sie den/die ursprünglichen Autor(en) und die Quelle ordnungsgemäß nennen, einen Link zur Creative Commons Lizenz beifügen und angeben, ob Änderungen vorgenommen wurden.

Die in diesem Artikel enthaltenen Bilder und sonstiges Drittmaterial unterliegen ebenfalls der genannten Creative Commons Lizenz, sofern sich aus der Abbildungslegende nichts anderes ergibt. Sofern das betreffende Material nicht unter der genannten Creative Commons Lizenz steht und die betreffende Handlung nicht nach gesetzlichen Vorschriften erlaubt ist, ist für die oben aufgeführten Weiterverwendungen des Materials die Einwilligung des jeweiligen Rechteinhabers einzuholen.

Weitere Details zur Lizenz entnehmen Sie bitte der Lizenzinformation auf http://creativecommons.org/ licenses/by/4.0/deed.de. 


\section{Literatur}

1. Baden LR, El Sahly HM, Essink B et al (2021) Efficacy and Safety of the mRNA-1273 SARS-CoV-2 Vaccine. NEngl J Med 384:403-416

2. Boyarsky BJ, Werbel WA, Avery RK et al (2021) Immunogenicity of a Single Dose of SARS-CoV-2 Messenger RNA Vaccine in Solid Organ Transplant Recipients. JAMA. https://doi.org/10.1001/jama. 2021.4385

3. D'agostino V, Caranci F, Negro A et al (2021) A rare case of cerebral venous thrombosis and disseminated Intravascular coagulation temporally associated to the COVID-19 vaccine administration. J Pers Med 11. https://doi.org/10. 3390/jpm11040285

4. Emary KRW, Golubchik T, Aley PK et al (2021) Efficacy of ChAdOx1 nCoV-19 (AZD1222) vaccine against SARS-CoV-2 variant of concern 202012/01 (B.1.1.7): an exploratory analysis of a randomised controlled trial. Lancet 397:1351-1362

5. Greinacher A, Thiele T, Warkentin TE et al (2021) Thrombotic thrombocytopenia after ChAdox 1 nCov-19 vaccination. NEnglJ Med. https://doi.org/ 10.1056/NEJMoa2104840

6. Herishanu Y, Avivil, Aharon A et al (2021) Efficacy of the BNT162b2 mRNA COVID-19 vaccine in patients with chronic lymphocytic leukemia. Blood. https:// doi.org/10.1182/blood.2021011568

7. Logunov DY, Dolzhikova IV, Shcheblyakov DV et al (2021) Safety and efficacy of an rAd26 and rAd5 vector-based heterologous primeboost COVID-19 vaccine: an interim analysis of a randomised controlled phase 3 trial in Russia. Lancet 397:671-681

8. Monin-Aldama L, Laing AG, Munoz-Ruiz $M$ et al (2021) Interim results of the safety and immuneefficacy of 1 versus 2 doses of COVID-19 vaccine BNT162b2 for cancer patients in the context of the UK vaccine priority guidelines. medRxiv. https:// doi.org/10.1101/2021.03.17.21253131

9. Oldenburg J, Klamroth R, Langer $F$ et al (2021) Diagnosis and management of vaccine-related thrombosis following AstraZeneca COVID-19 vaccination: guidance statement from the GTH. Hamostaseologie. https://doi.org/10.1055/a1469-7481

10. Polack FP, Thomas SJ, Kitchin N et al (2020) Safety and efficacy of the BNT162b2 mRNA Covid-19 vaccine. N Engl J Med 383:2603-2615

11. Rousseau B, Loulergue $P$, Mir $O$ et al (2012) Immunogenicity and safety of the influenza A H1N1v2009 vaccine in cancer patients treated with cytotoxic chemotherapy and/or targeted therapy: the VACANCE study. Ann Oncol 23:450-457

12. Rüthrich MM, Giessen-Jung $C$, Borgmann $S$ et al (2020) COVID-19 in cancer patients: clinical characteristics and outcome-an analysis of the LEOSS registry. Ann Hematol. https://doi.org/10. 1007/s00277-020-04328-4

13. Sahin U, Muik A, Derhovanessian E et al (2020) COVID-19 vaccine BNT162b1 elicits human antibody and $\mathrm{T}(\mathrm{H}) 1 \mathrm{~T}$ cell responses. Nature 586:594-599

14. Scully M, Singh D, Lown R et al (2021) Pathologic antibodies to platelet factor 4 after ChAdox $1 \mathrm{ncoV}$ 19 vaccination. N Engl J Med. https://doi.org/10. 1056/NEJMoa2105385

15. Sharma A, Bhatt NS, Martin StA et al (2021) Clinical characteristics and outcomes of COVID19 in haematopoietic stem-cell transplantation recipients: an observational cohort study. Lancet Haematol 8:e185-e193
16. Tiede A, Sachs UJ, Czwalinna A et al (2021) Prothrombotic immune thrombocytopenia after COVID-19 vaccine. Blood. https://doi.org/10.1182/ blood.2021011958

17. Vasileiou E, Simpson CR, Shi T et al (2021) Interim findings from first-dose mass COVID19 vaccination roll-out and COVID-19 hospital admissions in Scotland: a national prospective cohortstudy. Lancet 397:1646-1657

18. Voysey M, Costa Clemens SA, Madhi SA et al (2021) Safety and efficacy of the ChAdOx 1 nCoV-19 vaccine (AZD1222) against SARS-CoV-2: an interim analysis of four randomised controlled trials in Brazil, South Africa, and the UK. Lancet 397:99-111

19. Voysey M, Costa Clemens SA, Madhi SA et al (2021) Single-dose administration and the influence of the timing of the booster dose on immunogenicity and efficacy of ChAdOx1 nCoV-19 (AZD1222) vaccine: a pooled analysis of four randomised trials. Lancet 397:881-891

20. Wang Z, Schmidt F, Weisblum $Y$ et al (2021) mRNA vaccine-elicited antibodies to SARS-CoV-2 and circulating variants. Nature 592:616-622
Evidenz in der minimalinvasiven onkologischen Chirurgie

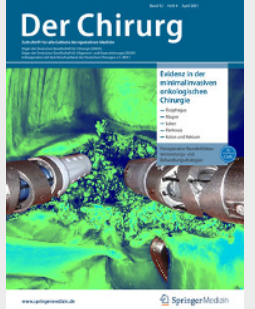

Die Einführung minimalinvasiver Techniken hat wie kaum eine andere Methode die operativen Eingriffe in der Allgemein- und Viszeralchirurgie revolutioniert. Der Chirurg 04/2021 bietet Ihnen Aktuelles zur Evidenz solcher Verfahren in der onkologischen Chirurgie. Bei der Auswahl der Autor*innen wurde besonders darauf geachtet, dass deren Arbeitsgruppen die genannten Methoden täglich in der eigenen Praxis anwenden. Damit ist gewährleistet, dass nicht nur aktuelle Literaturdaten, sondern auch individuelle Erfahrungen eingebracht wurden. Nutzen Sie die Orientierungsmöglichkeiten für die Wahl des Operationsverfahrens. Verschaffen Sie sich einen aktuellen Überblick über den Einsatz minimalinvasiver OPTechniken in der onkologischen Chirurgie für

$$
\begin{aligned}
& \text { - Ösophagus } \\
& \text { - Magen } \\
& \text { - Leber } \\
& \text { - Pankreas } \\
& \text { - Kolon } \\
& \text { - Rektum }
\end{aligned}
$$

Suchen Sie noch mehr zum Thema? Mit e.Med - den maßgeschneiderten Fortbildungsabos von Springer Medizin - haben Sie Zugriff auf alle Inhalte von SpringerMedizin.de. Sie können schnell und komfortabel in den für Sie relevanten Zeitschriften recherchieren und auf alle Inhalte im Volltext zugreifen.

Weitere Infos zu e.Med finden Sie auf springermedizin.de unter "Abos" 